Check for updates

Cite this: RSC Adv., 2018, 8, 29210

\section{Neuroprotective effect of total glycosides from paeonies against neurotoxicity induced by strychnos alkaloids related to recovering the levels of neurotransmitters and neuroendocrine hormones in rat serum and brain $\uparrow$}

\author{
Linjia Sun, ${ }^{a}$ Yu Chen, ${ }^{a}$ Chenzhi Hou, ${ }^{b}$ Xiaoyang Sun, ${ }^{a}$ Zhipeng Wang, ${ }^{a}$ Shujuan Li, ${ }^{a}$ \\ Mingming $L v^{a}$ and Xiaohui Chen (D)*a
}

\begin{abstract}
Semen Strychni, a classical traditional Chinese medicine, has been widely used for its anti-tumor, analgesic and anti-inflammatory angiogenesis effects. However, taking an overdose of Semen Strychni might result in extreme neurotoxicity. Strychnos alkaloids are the main toxic constituents of Semen Strychni. Total glycosides from paeonies are considered to have neuroprotective effects. In this study, twelve potential endogenous biomarkers in rat serum and brain were monitored to investigate the protective effect of total glycosides from the paeony against strychnos alkaloids-induced neurotoxicity. A sensitive liquid chromatography-tandem mass spectrometry method was developed and validated to monitor eight neurotransmitters including glutamate, $\gamma$-aminobutyric acid, acetylcholine serotonin, dopamine, norepinephrine, tryptophan and tyrosine. An enzyme-linked immunosorbent assay method was selected for determination of four neuroendocrine hormones including thyrotrophin-releasing hormone, corticotrophin-releasing hormone, antidiuretic hormone and prolactin. Results showed that continuous administration of strychnos alkaloids for 15 days caused significant changed levels of the biomarkers (especially the four neuroendocrine hormones). Meanwhile, total glycosides from paeony pretreated rats (administrated with total glycosides from the paeony for 15 days before exposure to strychnos alkaloids) showed recovered levels of these biomarkers. The results suggested that the neurotransmitters and neuroendocrine hormones in serum and brain might play potential roles as biomarkers. This study provides the possibility of alleviating the Semen Strychni-induced neurotoxicity in clinic by preprotection with total glycosides from paeonies.
\end{abstract}

Received 23rd June 2018

Accepted 9th August 2018

DOI: $10.1039 / \mathrm{c} 8 \mathrm{ra05384g}$

rsc.li/rsc-advances
Neurotransmitters are widely distributed in organisms, and play important roles in the nervous system's function. ${ }^{8}$ Abnormal alteration of neurotransmitters has been found to be closely associated with many neurological diseases including Parkinson's, Alzheimer's disease, depression and epilepsy. ${ }^{9}$ High level of glutamate (Glu), an excitatory amino acid, has been shown to cause damage of many cell components leading to neuronal death. ${ }^{\mathbf{1 0 , 1 1}}$ In contrast, $\gamma$-aminobutyric acid (GABA) is an inhibitory amino acid which can control the excitability and responsiveness of neurons. ${ }^{12}$ Acetylcholine (Ach) is supposed to activate the neuronal nicotinic acetylcholine receptors which could stimulate the voltage gated calcium channels, then causes accumulation of cytosolic calcium and triggers seizures. ${ }^{\mathbf{1 3 , 1 4}}$ Monoamine neurotransmitters including serotonin (5-HT), dopamine (DA) and norepinephrine (NE) are also found to be actively involved in the pathophysiology of drug-induced neurotoxicity. ${ }^{15-19}$ Tyrosine (Tyr) is the precursor of NE and DA, while tryptophan (Trp) is the precursor of 5-HT. Hence, the levels of these two amino acids could also reflect 
changes of monoamine neurotransmitters. As reported in some literature recently, the varied concentrations of neurotransmitters or their precursors may be the hallmark of nervous system abnormalities. ${ }^{20,21}$

Neuroendocrine hormones are associated with the nervous system function as well. Thyrotrophin-releasing hormone (TRH), corticotrophin-releasing hormone $(\mathrm{CRH})$ and antidiuretic hormone $(\mathrm{ADH})$ are peptide hormones which are firstly secreted by the neurosecretory cells of the hypothalamus and then drained via the pituitary portal system to their target cells. ${ }^{22}$ Some xenobiotics can damage the neurosecretory cells, leading to reduced synthesis and secretion of these hormones. ${ }^{23}$ It has been found that the concentrations of hypothalamic hormones had declined in some neurodegenerative diseases. ${ }^{24,25}$ Moreover, dysregulation of the hypothalamic-pituitary-adrenal axis is considered to be one of the major pathogenesis of depression. ${ }^{26}$ Prolactin (PRL), a protein hormone mainly synthesised and secreted by the lactogenic cells of the adenohypophysis, has multiple biological actions. ${ }^{27}$ Especially, it was indicated that PRL is involved in the brain recovery from hypoxic-ischemic injury. ${ }^{28}$ Based on these evidences it could be speculated that when herbinduced neurotoxicity happens, the metabolic equilibrium of hormones may have been disrupted, and levels of these hormones in serum and brain may alter.

As a common-used traditional medicine, Semen Strychni exhibits anti-tumor, analgesic, immune regulation, antiinflammatory angiogenesis and other therapeutic effects. ${ }^{29}$ However, for a long time, it was limited to use due to its fatal neurotoxicity. Alkaloids (e.g. strychnine and brucine), the main pharmacodynamic constituents of Semen Strychni, are the toxic constituents as well. ${ }^{30,31}$ Low doses of strychnos alkaloids increase the activity of certain functions in the central nervous system, while high doses will result in tonic convulsions and finally death through respiratory paralysis or cardiac arrest. ${ }^{32}$ In addition, the therapeutic dose of strychnos alkaloids is close to the toxic dose. ${ }^{33}$ For these reasons, it is urgent to alleviate the neurotoxicity caused by Semen Strychni and improve the application of Semen Strychni in clinic.

Total glycosides from paeony (TGP), mainly consist of paeoniflorin and albiflorin, are the extract of traditional Chinese medicine Radix Paeoniae Alba. According to literature, decocting together with Radix Paeoniae Alba simultaneously increased the therapeutic effects and the $\mathrm{LD}_{50}$ of Semen Strychni. ${ }^{34,35}$ Paeoniflorin and albiflorin have comprehensive pharmacological effects on nervous system, with a significant improvement in neurological diseases such as Alzheimer's, Parkinson's and cerebral ischemic injury. ${ }^{36-38}$ They are reported to have benefits like improving learning and memory, analgesia, conscious-sedation and antioxidation..$^{39,40}$ It has been demonstrated that TGP might exert its neuroprotective effect based on activating adenosine $\mathrm{A} 1$ receptor, regulating ion channel homeostasis, ameliorating the function of the cholinergic nerve, retarding oxidative stress and apoptosis of neurons and promoting nerve growth. ${ }^{41}$ In our previous study, we have found that TGP could improve the brain energy metabolism and rebuilds the homeostasis against strychnos alkaloids-induced neurotoxicity. ${ }^{42}$ Thus, we conjectured TGP to be a satisfactory protective drug against strychnos alkaloids-induced neurotoxicity.
In this study, eight neurotransmitters or their precursors (Glu, GABA, Ach, 5-HT, DA, NE, Trp and Tyr) in rat serum and brain were quantified simultaneously based on a liquid chromatography-tandem mass spectrometry (LC-MS/MS) method. Besides, enzyme-linked immunosorbent assay (ELISA) was selected for determination of four neuroendocrine hormones (TRH, CRH, ADH and PRL). The newly developed method was applied to investigate the neuroprotective effect of TGP against strychnos alkaloids-induced neurotoxicity. We not only aim to improve the clinical security of Semen Strychni, but also kindly expect our research will be helpful in monitoring the neurotoxicity caused by Semen Strychni or other herbs in early stages.

\section{Experimental}

\subsection{Materials and reagents}

Semen Strychni, Paeoniae Radix Alba and Datura stramonium (positive control herb) were obtained from Anguo Lengbei Co. Ltd (Baoding, Hebei, China). The herbs were authenticated by Professor Ying Jia (Pharmacognosy Department, Shenyang Pharmaceutical University). The reference standards of Glu, GABA, Ach, 5-HT, NE, DA, Trp, Tyr and tinidazole (IS) were purchased from Shanghai Yuanye Biological Technology Co., Ltd (Shanghai, China) (Fig. 1). The purities of the reference standards were all above $98 \%$. Acetonitrile and methanol (HPLC grade) were obtained from Fisher Scientific (Pittsburgh, USA). Formic acid was provided by Kermel Chemical Reagent Co., Ltd (Tianjin, China). Elisa kits of $\mathrm{TRH}, \mathrm{CRH}, \mathrm{ADH}$ and PRL were purchased from Shanghai Meilian Biological Technology Co., Ltd (Shanghai, China).

\subsection{Preparation of herbs}

The raw Semen Strychni was pulverized and extracted by refluxing with $50 \%$ ethanol $(1: 10, \mathrm{w} / \mathrm{v})$ for three times and $1 \mathrm{~h}$ for each time. The solutions were combined together, filtrated, and concentrated in vacuum to get the crude sample. Then it was suspended into water and regulated to $\mathrm{pH}=2$ with $\mathrm{HCl}$. The mixed solution was extracted with methylene chloride for four times. The aqueous layer was subsequently collected and alkalized to $\mathrm{pH}=10$ with $\mathrm{NaOH}$, then extracted with dichloromethane for another four times. The dichloromethane layer was concentrated by rotary evaporation then dried on water-bath. The resultant residue was the strychnos alkaloids extract (SAE).

Sliced Paeoniae Radix Alba was extracted three times with $70 \%$ ethanol $(1: 8, \mathrm{w} / \mathrm{v})$ for two hours each time. The extracts were obtained by concentrating the filtrates and drying under vacuum. Then the crude sample was suspended in water and chromatographed on macroporous resins with $70 \%$ ethanol. The eluate was collected and evaporated to dryness under reduced pressure, which yielded a dry powder of TGP.

Datura metel L. (mainly consists of scopolamine) was selected as positive control herb for its proved excited neurotoxicity. ${ }^{\mathbf{4 2 - 4 4}}$ The raw Datura metel L. was pulverized and extracted by refluxing with $75 \%$ ethanol $(1: 10, \mathrm{w} / \mathrm{v})$ for three times and $1 \mathrm{~h}$ for each time. The extract was obtained by concentrating the filtrates. Finally, all the extracts were dissolved with $0.5 \%$ carboxymethyl cellulose sodium (CMC-Na) for oral administration. 
<smiles>N[C@@H](CCC(=O)O)C(=O)O</smiles>

Glu<smiles>NCCc1c[nH]c2ccc(O)cc12</smiles>

5-HT<smiles>NC(Cc1c[nH]c2ccccc12)C(=O)O</smiles>

$\operatorname{Trp}$<smiles>NCCCC(=O)O</smiles>

GABA<smiles>NCCc1ccc(O)c(O)c1</smiles>

DA

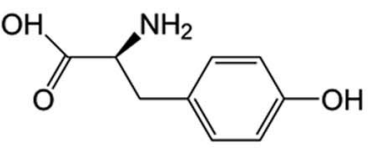

Tyr<smiles>CC(=O)OCC[N+](C)(C)C</smiles>

Ach<smiles>NCC(O)c1ccc(O)c(O)c1</smiles>

NE<smiles>CCS(=O)(=O)CCn1c([N+](=O)[O-])cnc1C</smiles>

Tinidazole(IS)

Fig. 1 Chemical structures of analytes and IS by LC-MS/MS.

\subsection{Animal treatment}

A total of 96 male Sprague-Dawley rats $(250 \pm 20 \mathrm{~g})$ were kindly provided by the Experimental Animal Central of Shenyang Pharmaceutical University. All animal experiments were strictly in accordance with the National Institutes of Health Guide for the Care and Use of Laboratory Animals, and were approved by the Institutional Ethical Committee (IEC) of Shenyang Pharmaceutical University. Animals were housed in laboratory conditions and acclimatized to 7 days before experiments.

In our previous studies, the following strategy to develop the neurotoxicity model was established and confirmed by behavioral tests and biochemical assay. ${ }^{45}$ Rats were randomly divided into four groups:

Blank group: rats were orally administrated with the vehicle (0.5\% CMC-Na solution) every day for 30 days.

Positive control group: rats were orally administrated with the vehicle (0.5\% CMC-Na solution) every day for 15 days, from 16th day were given Datura metel L. extract (equivalent to $14.78 \mathrm{~g}$ $\mathrm{kg}^{-1}$ calculated by raw herb) every day for 15 days.

Strychnos alkaloids extract (SAE) group: rats were orally administrated with the vehicle (0.5\% CMC-Na solution) every day for 15 days, from 16 th day were given $\operatorname{SAE}\left(12 \mathrm{mg} \mathrm{kg}^{-1}\right)$ for 15 days.

Total glycosides from paeony (TGP) group: rats were orally administrated with TGP (250 $\mathrm{mg} \mathrm{kg}^{-1}$ ) every day for 15 days, and from 16th day were given SAE (12 $\left.\mathrm{mg} \mathrm{kg}^{-1}\right)$ for 15 days.

\subsection{Bio-sample collection}

Blood samples were collected $0.5 \mathrm{~h}$ after oral administration on the 15th, 16th (the first day of orally administrating SAE and Datura metel L.), 18th, 23rd, 30th day, and serum was obtained by centrifugation of blood (3000 rpm for $8 \mathrm{~min})$. Rats $(n=6$ for each group) were separately sacrificed on the 16th, 18th, 23rd, 30th day then the brain tissues were excised. All the samples were stored at $-80{ }^{\circ} \mathrm{C}$ until analysis.

On the 30th day after administration, brain samples for histopathology were collected and fixed in $10 \%$ formalin-saline for $48 \mathrm{~h}$, then embedded in paraffin.

\subsection{Histopathology}

Brain samples were sliced to $3 \mu \mathrm{m}$ wax sections, and the tissue sections were stained with hematoxylin and eosin (H\&E) for microscopic examinations after gradual rehydration in a series of graded alcohols.

\subsection{LC-MS/MS assay of neurotransmitters and their precursors}

2.6.1 Instrument and conditions. An AB SCIEX Instruments 5500 triple quadrupole (Applied Biosystems, Foster City, CA) interfaced online with an Agilent 1260 Series Rapid Resolution LC system (Agilent Technologies, Santa Clara, CA) was applied in the assay. The chromatographic separation was achieved on a Shiseido CAPCELL PAK $\mathrm{C}_{18}$ MG column $(4.6 \mathrm{~mm}$ $\times 150 \mathrm{~mm}, 5 \mu \mathrm{m}$ ). A water (containing $0.1 \%$ formic acid, solvent A)/acetonitrile (solvent B) system was used as the mobile phase. Separation was completed using the following gradient

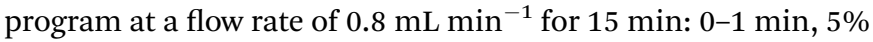
B; 1-10 min, 5-95\% B; 10-11 min, 95\% B; 11-12 min, 95-5\% B; 12-15 min, $5 \%$ B. The column temperature was set at $30^{\circ} \mathrm{C}$.

An electrospray ionisation (ESI) interface and triple quadruple mass analyser was used in this method. The analytes and IS were analyzed by multiple-reaction monitoring (MRM) scanning in a positive ion mode. The optimized mass spectrometric instrument parameters obtained after tuning were as follows: ion source temperature was at $650{ }^{\circ} \mathrm{C}$; curtain gas, gas 1 and gas 2 were 35, 50 and 50 psi; ion spray voltage and entrance 
potential were 5500 and $10 \mathrm{~V}$, respectively. The characteristics of ion pairs (corresponding to declustering potential DP, collision energy CE, collision cell exit potential CXP) were $m / z 148.2 \rightarrow$ $84.1(40,24,16)$ for glutamate, $m / z 104.2 \rightarrow 87.1(24,15,18)$ for $\gamma$-aminobutyric acid, $m / z 146.2 \rightarrow 87.2(49,20,5)$ for acetylcholine, $m / z 177.1 \rightarrow 160.2(41,15,11)$ for serotonin, $m / z 154.1$ $\rightarrow 137.2(38,14,8)$ for dopamine, $m / z 170.1 \rightarrow 152.2(32,11,8)$ for norepinephrine, $m / z 182.0 \rightarrow 136.1(50,16,8)$ for tyrosine, $m / z 205.2 \rightarrow 188.0(41,15,11)$ for tryptophan and $m / z 248.1 \rightarrow$ $121.2(72,24,25)$ for tinidazole (IS).

2.6.2 Preparation of calibration standard and quality control (QC) samples. The standard stock solutions $(1.0 \mathrm{mg}$ $\mathrm{mL}^{-1}$ ) of Glu, GABA, Ach, 5-HT, NE, DA, Trp, and IS were separately prepared in methanol-water $(70: 30, \mathrm{v} / \mathrm{v})$. Tyr was prepared in methanol-water $(10: 90, \mathrm{v} / \mathrm{v})$ containing $0.5 \% 0.5 \mathrm{M}$ hydrochloric acid. The stock solutions were further diluted with methanol to make a series of mixed working solutions.

Calibration standards of analytes were prepared by spiking blank serum or brain homogenate $(100 \mu \mathrm{L})$ into working standards $(100 \mu \mathrm{L})$ at different concentrations which were evaporated to dryness at $30{ }^{\circ} \mathrm{C}$ under a stream of nitrogen before sample preparation. The calibration ranges of analytes are shown in Tables S1 and S2. $\uparrow$ The QC samples were prepared in the same way at low, middle and high concentrations for serum and brain homogenate as shown in Tables S3-S6. $\dagger$

2.6.3 Sample preparation. All frozen samples were thawed at room temperature prior to analysis. An aliquot of $100 \mu \mathrm{L}$ serum was spiked with $10 \mu \mathrm{L}$ IS $\left(0.5 \mu \mathrm{g} \mathrm{mL}{ }^{-1}\right)$ and vortex-mixed for $30 \mathrm{~s}$, then deproteinized with $300 \mu \mathrm{L}$ acetonitrile by vortexing for $5 \mathrm{~min}$. After centrifugation (13000 rpm, $5 \mathrm{~min}$ ), $100 \mu \mathrm{L}$ of supernatants were diluted to $500 \mu \mathrm{L}$ with acetonitrile. An aliquot of $10 \mu \mathrm{L}$ of the solution was injected into the LC-MS/MS system for analysis.
The brain tissue samples were homogenized in acetonitrile $(1: 10, \mathrm{w} / \mathrm{v})$ containing $0.1 \%$ formic acid. Every $100 \mu \mathrm{L}$ of homogenate was spiked with $10 \mu \mathrm{L}$ IS $\left(1 \mu \mathrm{g} \mathrm{mL}{ }^{-1}\right)$ and vortexmixed for $30 \mathrm{~s}$, then the mixtures were extracted by protein precipitation using $890 \mu \mathrm{L}$ acetonitrile. After vortexing for $5 \mathrm{~min}$ and centrifugation at $13000 \mathrm{rpm}$ for $5 \mathrm{~min}$ at $4{ }^{\circ} \mathrm{C}, 10 \mu \mathrm{L}$ of the supernatants were injected for analysis.

2.6.4 Method validation. The method was validated according to the US Food and Drug Administration bioanalytical method validation guidances with respect to lower limit of quantification (LLOQ), linearity, precision, accuracy, absolute recovery, matrix effect and stability. ${ }^{46}$ Because of the inherent existence of these endogenous substances in the serum and brain, blank value of each analyte should be subtracted from each calibration point.

\subsection{ELISA assay of neuroendocrine hormones}

$\mathrm{CRH}, \mathrm{TRH}, \mathrm{ADH}$ and PRL were determined using ELISA kits in conformity with the directions of the manufacturer in 96-well plates. The brain tissue samples were homogenized in ten-fold volume of phosphate buffer solution $(\mathrm{pH}=7.4)$ then centrifugated (3000 rpm, $20 \mathrm{~min}$ ). The supernatants were collected for determination. The serum samples were used directly. After the enzyme reaction, the absorbance was determined at $450 \mathrm{~nm}$ using microplate reader F039003 (TECAN, Austria), and the concentrations were calculated according to the standard curve, which were in the ranges of $0.5-8 \mu \mathrm{IU} \mathrm{mL}^{-1}$ for TRH, 5-80 $\mathrm{ng} \mathrm{L}^{-1}$ for CRH, 5-80 ng $\mathrm{L}^{-1}$ for $\mathrm{ADH}$ and $50-800 \mathrm{ng} \mathrm{L}^{-1}$ for PRL.

\subsection{Data analysis and statistics}

Statistical data were processed using SPSS (version 19.0). Differences between different groups were evaluated by one-way
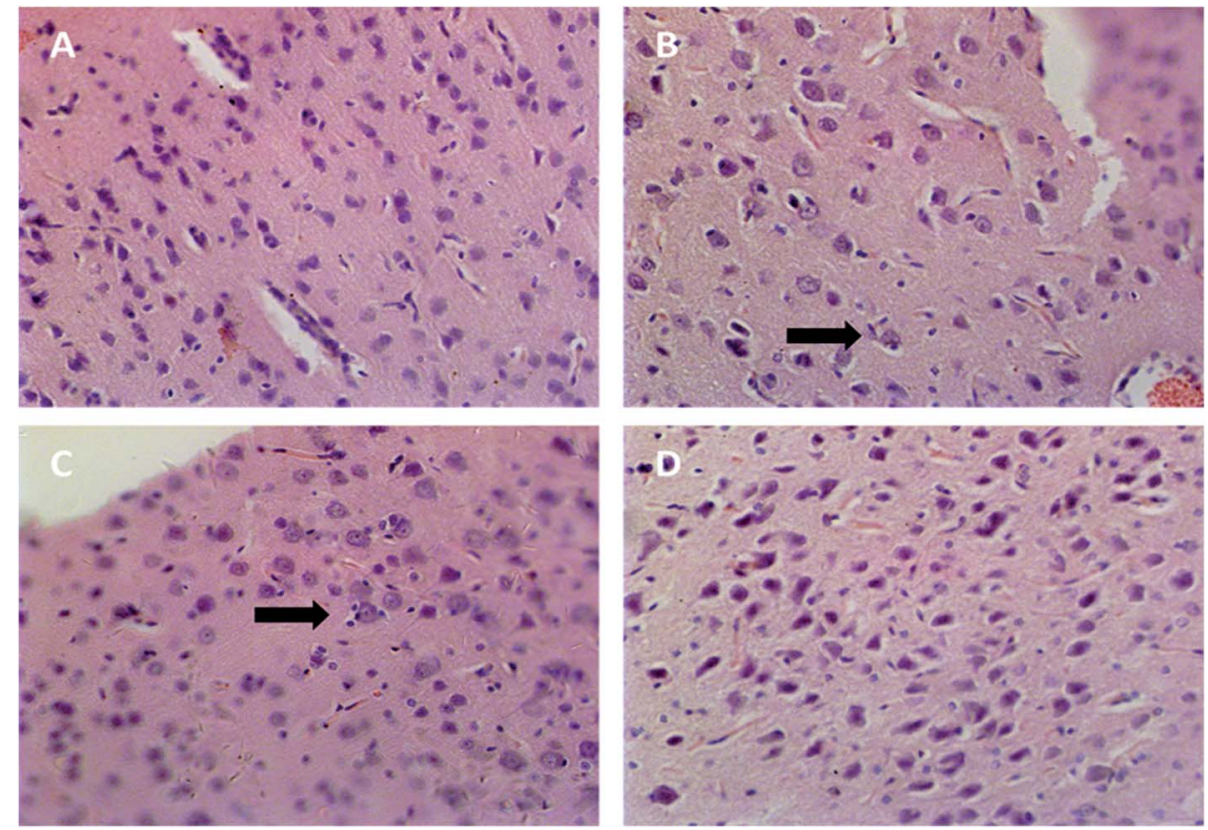

Fig. 2 Representative histopathological photographs of rat cerebral cortex $(40 \times)$. (A) The blank group, (B) the positive control group, (C) SAE group, (D) TGP group. Arrow: neuronophagia. 
analysis of variance (ANOVA) followed by LSD multiple comparison tests. Statistical significance was considered when the value of $p$ less than 0.05 or 0.01 .

\section{Results and discussion}

\subsection{Histopathology}

As shown in Fig. 2, the brain tissue specimen in the blank group showed normal appearances. In the positive control group and SAE group, the specimens showed severe neuronophagia at cerebral cortex, indicating the necrosis of neurons. The specimen in TGP group showed normal appearances compared with the SAE group, except for mild hyperplasia of the microglia.

\subsection{Method validation of LC-MS/MS}

The retention times of Glu, GABA, Ach, 5-HT, DA, NE, Trp, Tyr and IS in samples were 1.92, 1.87, 2.01, 5.54, 3.10, 1.93, 6.40, 3.69 and 7.62 min respectively (Fig. 3), which were in accordance with those of standard solutions. The calibration curves of analytes were constructed by weighted $\left(1 / x^{2}\right)$ least square linear regression. LLOQ was defined as the lowest concentration of the calibration curve with acceptable accuracy and precision. Good linearities with $r>0.9904$ and LLOQ in serum and brain are showed in Tables S1 and S2. $\dagger$ The precision and accuracy of the method were evaluated by six replicates at each QC level per day for three days. The intra-day and inter-day precision (RSD, $\%$ ) were less than $13.9 \%$ and the accuracy (RE, \%) ranged from $-4.6 \%$ to $5.6 \%$. The mean recovery was more than $73.2 \%$ at each concentration and the matrix effects ranged from $91.0 \%$ to $110.0 \%$. The stability of these analytes at room temperature for $4 \mathrm{~h},-80{ }^{\circ} \mathrm{C}$ for 1 month, freeze-thaw cycles, and $4{ }^{\circ} \mathrm{C}$ in the auto-sampler for $12 \mathrm{~h}$ were all within the acceptable range. (Tables S3-S6 $\dagger$ ). These results indicated that the developed method was reliable and reproducible for quantitative determination.

\subsection{Neurotoxicity biomarkers assays}

The column chart of the levels of neurotransmitters in serum is shown in Fig. 4. When compared with the blank group, the level of Ach in rat serum of positive control and SAE group showed a significant elevation since the 18th day $(p<0.05)$; the levels of 5 -HT and Trp in serum statistically increased from the 23rd day $(p<0.05)$; the concentrations of DA, NE and Tyr showed significant reduction since the 23rd day $(p<0.05)$; the level of Glu increased slightly and the level of GABA decreased slightly. While, the levels of analytes almost returned to normal levels in
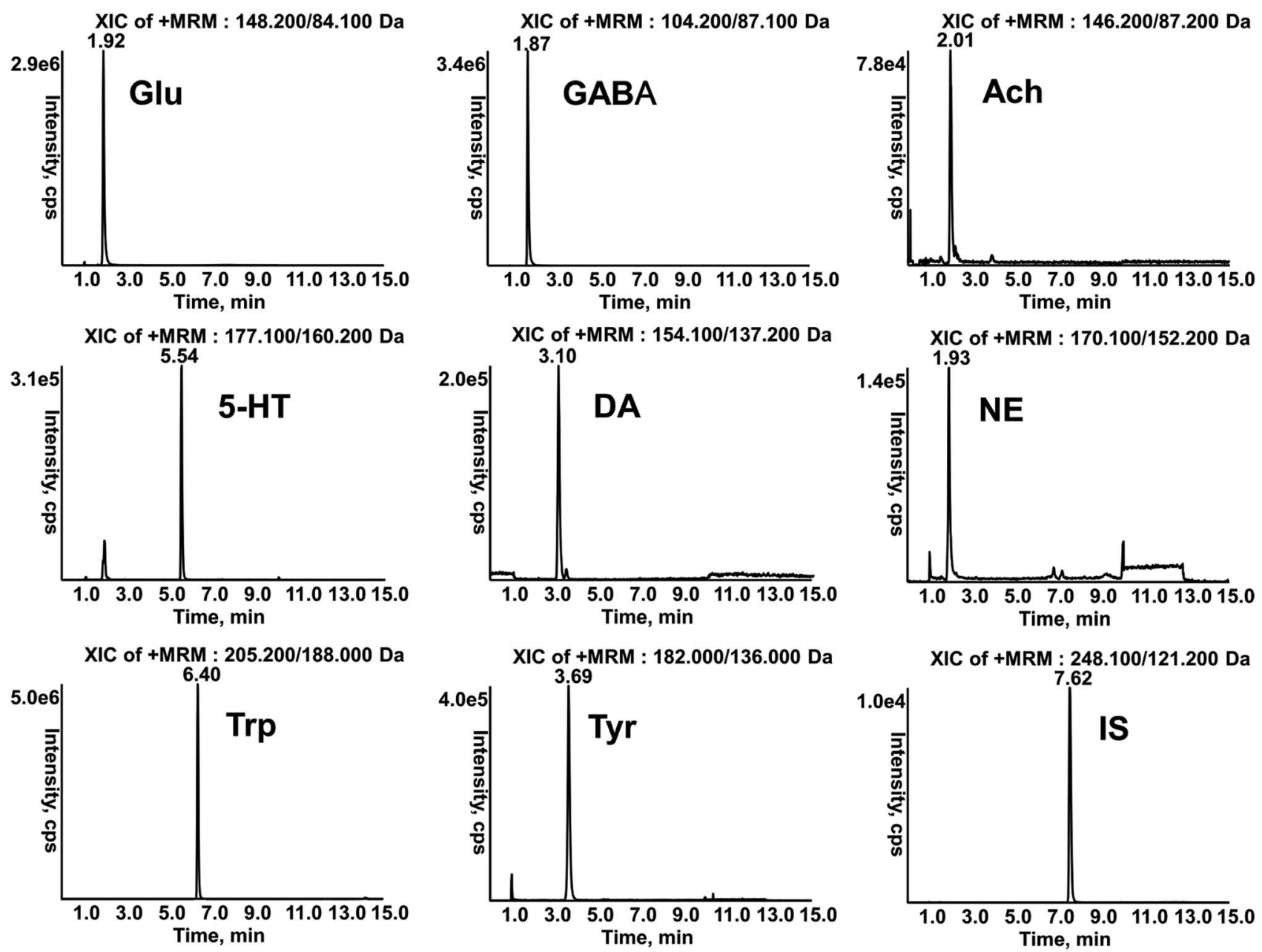

Fig. 3 Representative chromatograms of profiles of analytes by LC-MS/MS. 

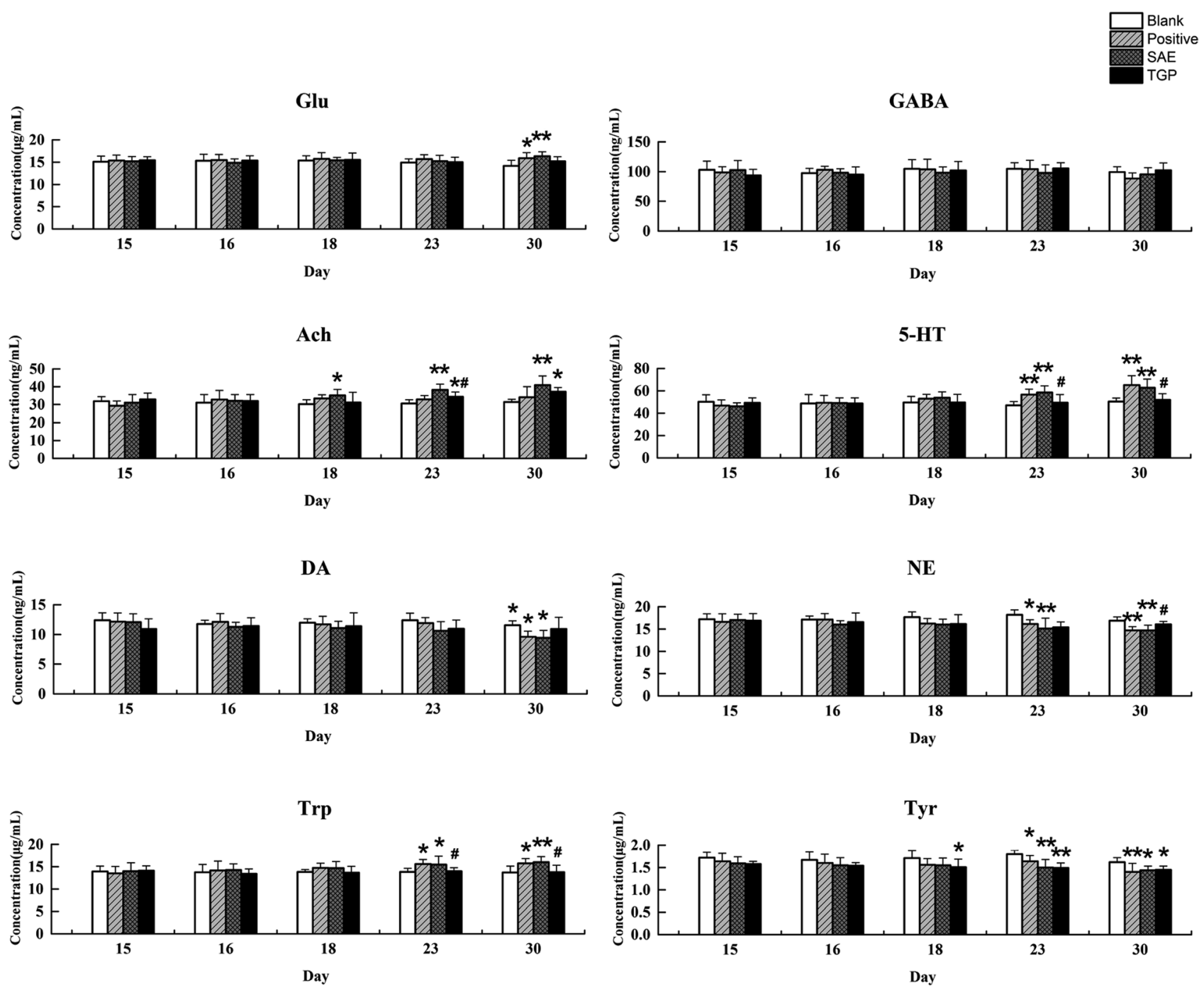

Fig. 4 Results of levels of Glu, GABA, Ach, 5-HT, DA, NE, Trp and Tyr in serum of four groups on different days by LC-MS/MS ( $n=6)$. ** $p<0.01$ and $* p<0.05$ (compared with blank group rats); $\# p<0.01$ and ${ }^{\#} p<0.05$ (compared with SAE group rats).

the TGP group when comparing to the SAE group $(p<0.05)$. The levels of neurotransmitters in brain are shown in Fig. 5 , the concentration trends of these biomarkers in brain were generally in agreement with those in serum.

As to neuroendocrine hormones, compared with the concentrations in the blank group, levels of TRH, CRH, ADH and PRL in the positive control and SAE group showed an obvious decreasing trend in serum since the 16th day $(p<0.05)$, as shown in Fig. 6 . The TGP group showed a significant recovery towards normal levels when compared with the SAE group. Fig. 7 shows the levels of neuroendocrine hormones in brain samples. Similar as the serum samples, the concentrations in the positive control and SAE group decreased significantly since the 16th day ( $p<0.05$ ), except for TRH (the 23rd day). Meanwhile, the TGP group showed a significant difference when compared with the SAE group. The above results showed that these neurotransmitters and hormones metabolic pathways might be disturbed after administration of SAE, and TGP could recover them significantly.

\subsection{Biochemical interpretation}

The doses (12 $\mathrm{mg} \mathrm{kg}^{-1}$ for SAE, $14.78 \mathrm{~g} \mathrm{~kg}^{-1}$ for Datura metel L. and $250 \mathrm{mg} \mathrm{kg}^{-1}$ for TGP) were selected based on our previous study, to achieve the most appropriate toxic and pre-protect effects. $^{45}$ In this study, histopathological results revealed a high dose of SAE might cause brain injury by successive administration for 15 days. Moreover, pretreatment of TGP for 15 days could attenuate this damage. Levels of twelve analytes changed significantly after successive administration of strychnos alkaloids, in accordance with alterations in the positive control group, indicating their potential roles of neurotoxicity biomarkers.

Strychnine and brucine take up more than $70 \%$ among the strychnos alkaloids. ${ }^{47}$ Although strychnine and brucine are difficult to cross the blood-brain barrier, powerful central nervous excitement effect could be exhibited. ${ }^{48}$ Firstly, strychnine blocks or antagonizes the inhibitory glycine receptor (GlyR), a ligand-gated chloride channel in the spinal cord. ${ }^{49}$ The 

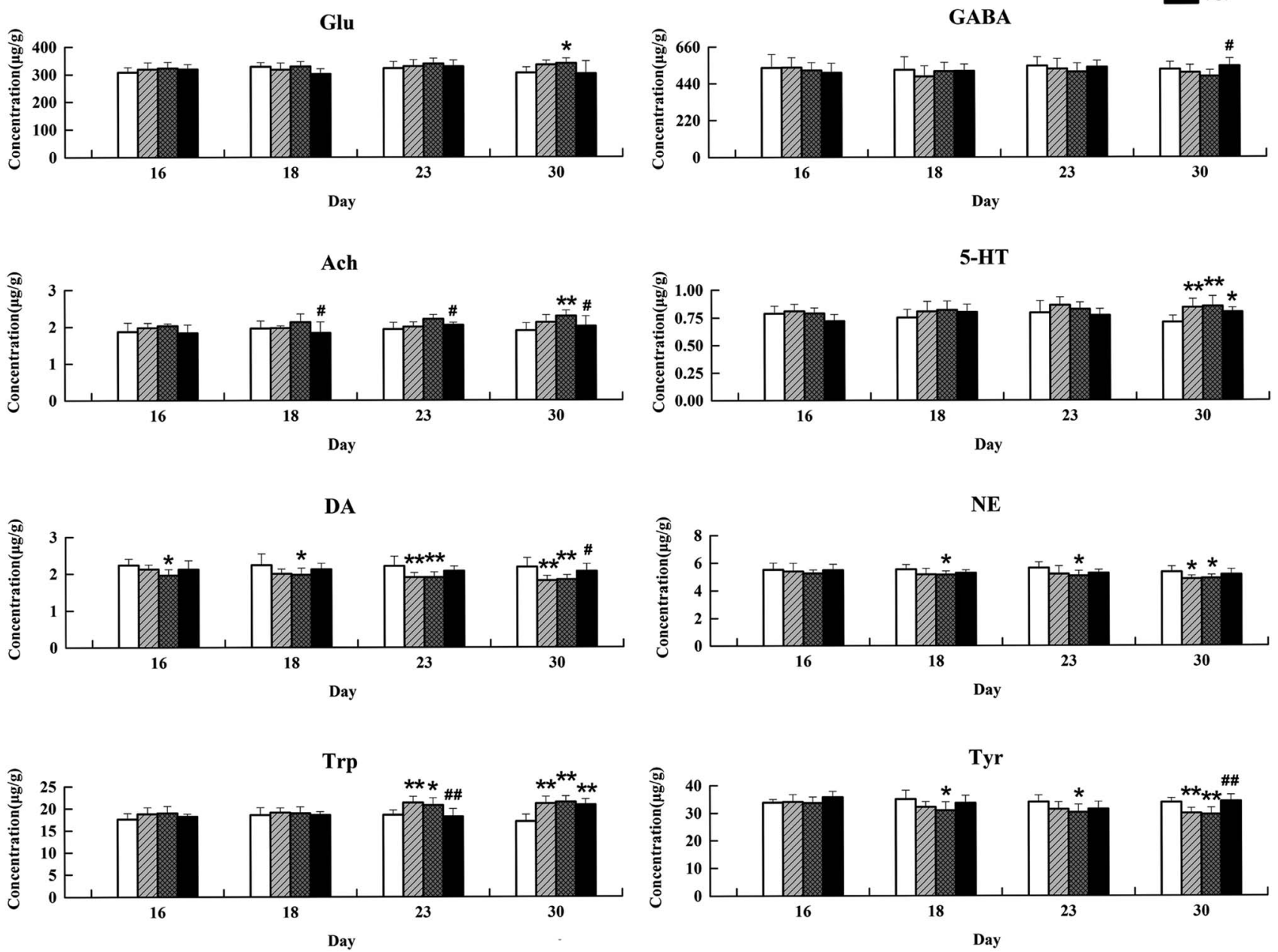

Fig. 5 Results of levels of Glu, GABA, Ach, 5-HT, DA, NE, Trp and Tyr in brain tissue of four groups on different days by LC-MS/MS ( $n=6)$. ** $p<$ 0.01 and $* p<0.05$ (compared with blank group rats); ${ }^{\# \#} p<0.01$ and ${ }^{\#} p<0.05$ (compared with SAE group rats).

excitatory synapses can then exert their action unrestrainedly, which might cause an increase in spinal reflex. ${ }^{32}$ At the same time, whole central nervous system including the medullary respiratory center, vascular motor center and the cerebral cortex sensory center might be irritated. ${ }^{47,50,51}$ Thus, a high dose of strychnine can induce central or peripheric excitatory reactions like hyperactivity of sensory, dyspnoea, tonic convulsions and finally death through respiratory or spinal paralysis or by cardiac arrest. Toxicity of brucine is similar to that of strychnine, but the toxic dose of brucine is much higher than strychnine. ${ }^{32}$

The balance between excitation and inhibition in the nervous system is related to multiple neurotransmitters. As an example, glutamate, acetylcholine and serotonin are excitatory neurotransmitter, while dopamine and GABA belong to inhibitory neurotransmitter. ${ }^{52}$ Serotonergic, noradrenergic, dopaminergic, cholinergic GABAergic and glutamatergic neural circuitry interact with each other to maintain the normal activity of the nervous system. ${ }^{53}$ Exposure to neurotoxicants can alter the synthesis, store and release of some neurotransmitters, which might cause many abnormal physiological states. Based on results in this study, a speculation could be established that the strychnos alkaloids do not directly damage the brain, but lead lesion through the over-excitement of CNS which could be reflected by the disordered neurotransmission.

Neuroendocrine hormones have comprehensive regulatory effect on CNS. Feedback regulation exists between neurotransmitters and neuroendocrine hormones, which might be implicated in some neurodegenerative diseases or cerebral trauma. ${ }^{54-56}$ This study demonstrated that the neurotoxicity induced by strychnos alkaloids could cause the decrease of TRH, CRH, ADH and PRL in serum and brain. It could be inferred that excessive excitement by strychnos alkaloids could induce degeneration or death of hypothalamic and pituitary neurosecretory cells then damage the hypothalamic-pituitarygland axis, causing decreased secretion of hormones. ${ }^{57}$

Pre-administration of TGP significantly recovered the changed biomarkers induced by strychnos alkaloids in this study. Strychnine and brucine are substrates of CYPs. ${ }^{58}$ It was reported that paeoniflorin and albiflorin (main compounds of TGP) could regulate the activity of CYP3A4 and CYP2D6. ${ }^{59}$ 


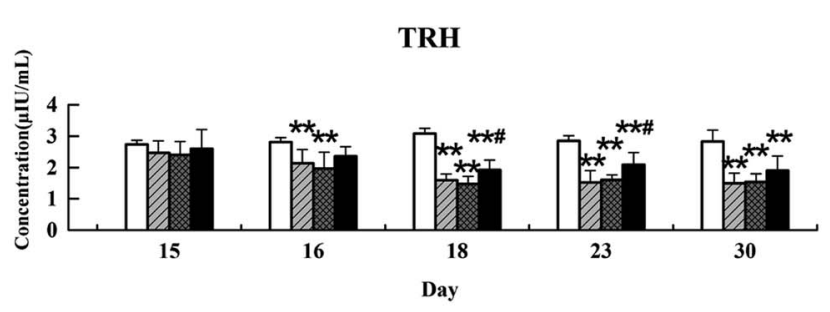

ADH

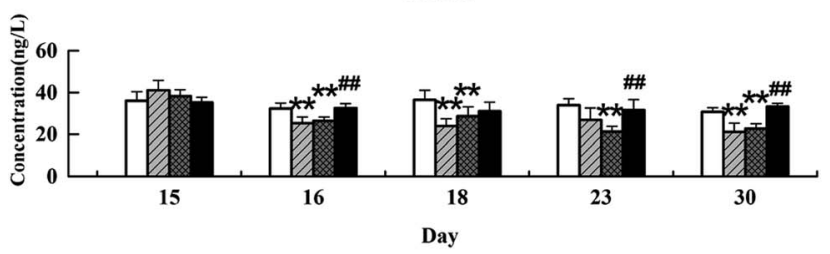

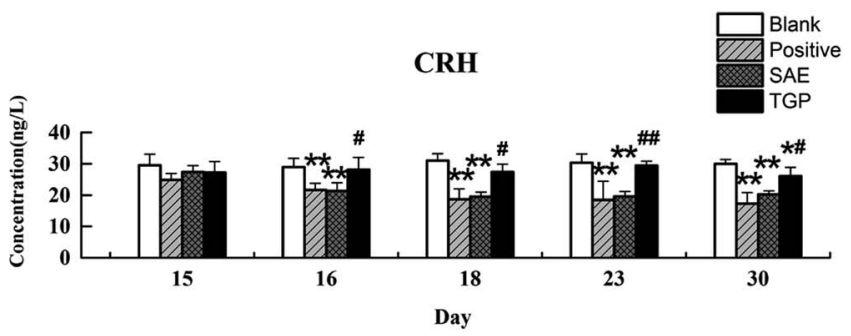

PRL

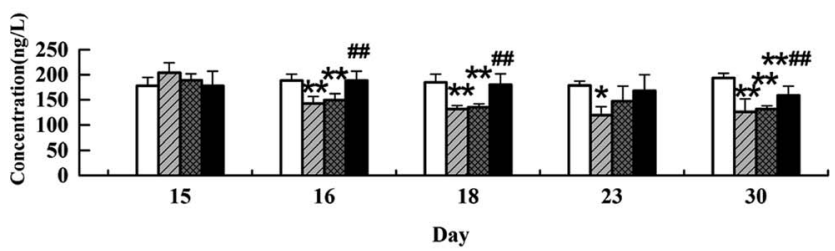

Fig. 6 Results of levels of TRH, CRH, ADH and PRL in serum of four groups on different days by ELISA $(n=6)$. **p $p<0.01$ and *p<0.05 (compared with blank group rats); ${ }^{\# \#} p 0.01$ and ${ }^{\#} p<0.05$ (compared with SAE group rats).

Previous toxicokinetics study suggested that TGP delayed the absorption and accelerated the metabolism of strychnine and brucine, thus reduced the neurotoxicity. ${ }^{60}$ That might interpret the normalization of biomarkers in the TGP group in some ways. For another hand, paeoniflorin and albiflorin have comprehensive neuroprotective effects on nervous system. These effects have been attributed to the involvement of multiple modulatory pathways, mainly anti-oxidative stress, regulating calcium ion homeostasis, anti-inflammatory and inhibiting cell apoptosis. ${ }^{61}$ TGP was reported to possess the ability to penetrate the blood-brain barrier, indicating the possibility that TGP can directly act on the CNS. ${ }^{62}$ Paeoniflorin could exert neuroprotective effects by activating adenosine A1 receptor, which was widely distributed in the central nervous system. ${ }^{63}$ Adenosine A1 receptor may have mediating effects on neurotransmitters release, such as Ach and DA, to regulate the excitation-inhibition balance. ${ }^{64}$ Paeoniflorin and albiflorin also have the potential to promote neuron growth and prevent neuron damage. ${ }^{65}$ From the results, TGP could restore the secretory dysfunction of neurosecretory cells caused by strychnos alkaloids. The accumulation of neuroprotective effects could antagonize cellular damage and imbalance of central nervous system induced by strychnos alkaloids, which could be reflected by the concentrations of neurotransmitters and hormones. However, further studies are required to elucidate the underlying molecular mechanisms.

According to the results, the neurotransmitters and hormones are able to be regarded as biomarkers of strychnos alkaloids-induced neurotoxicity. The results of this study showed that TGP could significantly alleviate the neurotoxic effect of strychnos alkaloids. This study, together with previous studies, provided the evidences for the interventional effects of TGP against strychnos alkaloids-induced neurotoxicity and possibility of clinical pretreatment.
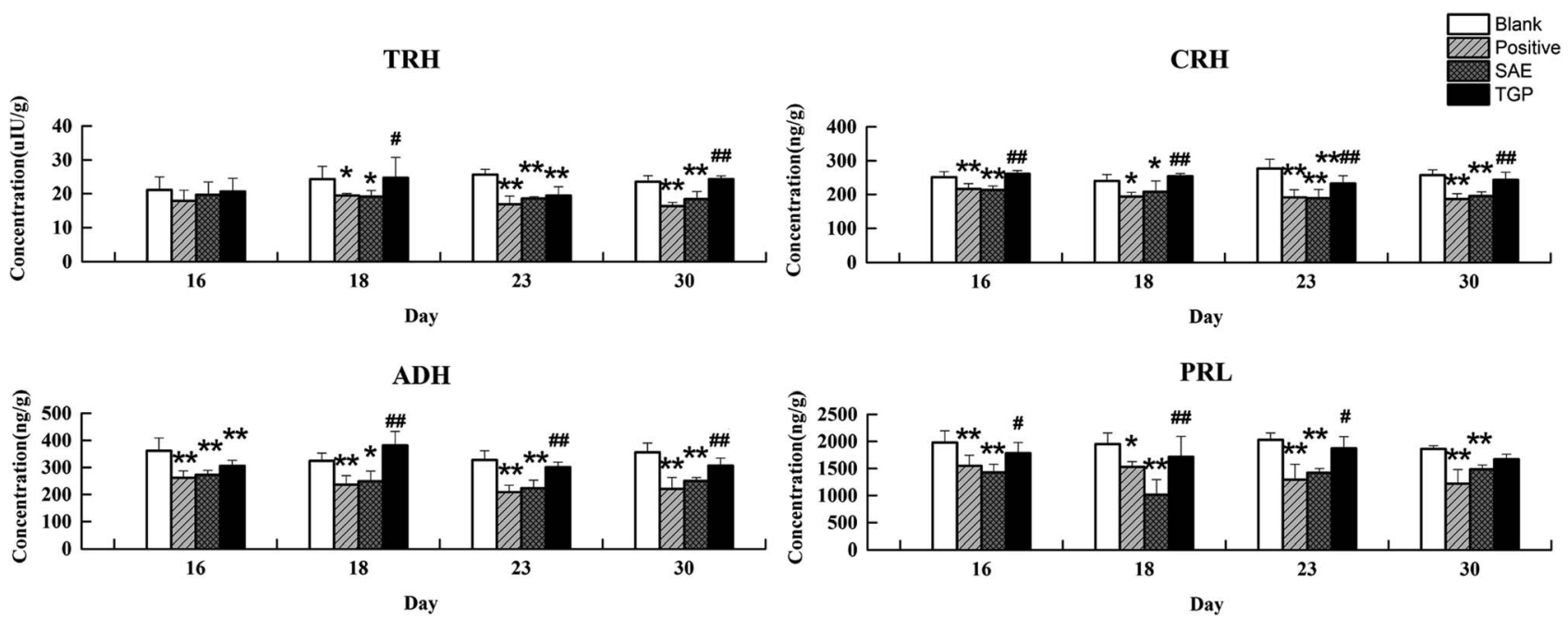

Fig. 7 Results of levels of TRH, CRH, ADH and PRL in brain tissue of four groups on different days by ELISA $(n=6)$. ** $p<0.01$ and $* p<0.05$ (compared with blank group rats); ${ }^{\# \#} p<0.01$ and ${ }^{\#} p<0.05$ (compared with SAE group rats). 


\section{Conclusions}

In the present study, the protective effect of TGP against neurotoxicity induced by strychnos alkaloids was evaluated through monitoring the levels of neurotransmitters and neuroendocrine hormones comprehensively. The results indicated that pretreatment with TGP could recover the levels of these biomarkers, regulate homeostasis, and thus exert neuroprotective effect against neurotoxicity induced by strychnos alkaloids. This investigation may not only be applied to diagnose neurotoxicity induced by strychnos alkaloids or other herbs, but also valuable for alleviating the Semen Strychniinduced neurotoxicity in clinic by pre-protection with TGP.

\section{Conflicts of interest}

The authors have declared no conflict of interest.

\section{Abbreviations}

$\begin{array}{ll}\text { Glu } & \text { Glutamate } \\ \text { GABA } & \gamma \text {-Aminobutyric acid } \\ \text { Ach } & \text { Acetylcholine } \\ \text { 5-HT } & \text { Serotonin } \\ \text { DA } & \text { Dopamine } \\ \text { NE } & \text { Norepinephrine } \\ \text { Tyr } & \text { Tyrosine } \\ \text { Trp } & \text { Tryptophan } \\ \text { TRH } & \text { Thyrotrophin-releasing hormone } \\ \text { CRH } & \text { Corticotrophin-releasing hormone } \\ \text { ADH } & \text { Antidiuretic hormone } \\ \text { PRL } & \text { Prolactin } \\ \text { TGP } & \text { Total glycosides from paeony } \\ \text { LC-MS/ } & \text { Liquid chromatography-tandem mass spectrometry } \\ \text { MS } & \\ \text { ELISA } & \text { Enzyme-linked immunosorbent assay } \\ \text { IS } & \text { Internal standard } \\ \text { SAE } & \text { Strychnos alkaloids extract } \\ \text { CNS } & \text { Central nervous system }\end{array}$

\section{Acknowledgements}

This work was financially supported by Natural Science Foundation of China (No. 81673577).

\section{References}

1 Y. Zhao, Z. Zuo, X. Liu and Y. Sun, Chin. Tradit. Herb. Drugs, 2011, 42, 392-397.

2 X. Xu, W. Li, S. Mi and N. Wang, Chin. J. Pharmacov., 2011, 8, 678-680.

3 L. Schultz, M. G. Zurich, M. Culot, A. da Costa, C. Landry, P. Bellwon, T. Kristl, K. Hormann, S. Ruzek, S. Aiche, K. Reinert, C. Bielow, F. Gosselet, R. Cecchelli, C. G. Huber, O. H. Schroeder, A. Gramowski-Voss,
D. G. Weiss and A. Bal-Price, Toxicol. In Vitro, 2015, 30, 138-165.

4 C. Iadecola, K. Niwa, S. Nogawa, X. Zhao, M. Nagayama, E. Araki, S. Morham and M. E. Ross, Proc. Natl. Acad. Sci. U. S. A., 2001, 98, 1294.

5 S. D. Yan, X. Chen, J. Fu, M. Chen, H. Zhu, A. Roher, T. Slattery, L. Zhao, M. Nagashima and J. Morser, Nature, 1996, 382, 685-691.

6 M. Y. Xu, Y. J. Sun, P. Wang, H. Y. Xu, L. P. Chen, L. Zhu and Y. J. Wu, Chem. Res. Toxicol., 2015, 28, 1216-1223.

7 H. Zetterberg, Neurological Biomarkers, Wiley-VCH Verlag GmbH \& Co. KGaA, 2016.

8 S. Bourcier, J. F. Benoist, F. Clerc, O. Rigal, M. Taghi and Y. Hoppilliard, Rapid Commun. Mass Spectrom., 2006, 20, 1405-1421.

9 S. Nakamura, Nihon Rinsho, 1992, 50, 1637.

10 A. Atlante, P. Calissano, A. Bobba, S. Giannattasio, E. Marra and S. Passarella, FEBS Lett., 2001, 497, 1.

11 D. Ramonet, M. J. Rodríguez, K. Fredriksson, F. Bernal and N. Mahy, Hippocampus, 2004, 14, 586.

12 D. A. Mccormick, J. Neurophysiol., 1989, 62, 1018.

13 C. Cardone, J. Szenohradszky, S. Yost and P. E. Bickler, Anesthesiology, 1994, 80, 1155-1161; discussion 1129A.

14 A. K. Rehni and T. G. Singh, Naunyn Schmiedebergs Arch. Pharmacol., 2010, 382, 279-285.

15 C. Maximino, J. Araujo, L. K. R. Leão, A. B. A. Grisolia, K. R. M. Oliveira, M. G. Lima, E. d. J. O. Batista, M. E. Crespo-López, A. Gouveia and A. M. Herculano, Neurotoxicol. Teratol., 2011, 33, 727-734.

16 R. Joseph, C. Tsering, S. Grunfeld and K. M. A. Welch, Neurosci. Lett., 1992, 136, 15-18.

17 D. M. Thomas, M. Angoa Perez, D. M. Francescutti-Verbeem, M. M. Shah and D. M. Kuhn, J. Neurochem., 2010, 115, 595605.

18 S. Choudhary, G. Raheja, V. Gupta and K. D. Gill, J. Biochem., Mol. Biol. Biophys., 2002, 6, 29-36.

19 S. Singh, S. Jamwal and P. Kumar, Neurochem. Res., 2015, 40, 1758-1766.

20 H. Xing, K. Zhang, R. Zhang, Y. Zhang, L. Gu, H. Shi, K. Bi and X. Chen, J. Chromatogr. B: Anal. Technol. Biomed. Life Sci., 2015, 988, 135-142.

21 B. Wei, Q. Li, R. Fan, D. Su, X. Chen, Y. Jia and K. Bi, J. Pharm. Biomed. Anal., 2014, 88, 416-422.

22 G. M. Besser and C. H. Mortimer, J. Clin. Pathol., 1974, 27, 173-184.

23 M. Kawahara, M. Kato-Negishi and Y. Kuroda, Cell. Mol. Neurobiol., 2002, 22, 87-93.

24 A. C. Reid and J. J. Morton, J. Neurol. Sci., 1982, 54, 295-301. 25 P. S. Sørensen, M. Hammer, S. Vorstrup and F. Gjerris, J. Neurol., Neurosurg. Psychiatry, 1983, 46, 911-916.

26 E. J. Nestler, M. Barrot, R. J. Dileone, A. J. Eisch, S. J. Gold and L. M. Monteggia, N. Engl. J. Med., 1989, 320, 869-870.

27 M. E. Freeman, B. Kanyicska, A. Lerant and G. Nagy, Physiol. Rev., 2000, 80, 1523-1631.

28 M. d. Tae, Protection and recovery of the injured brain the significance of the cerebral growth hormone and prolactin axes, University of Auckland, 2006. 
29 L. Gu, S. Li, R. Zhang, Y. Zhang, X. Wang, K. Zhang, Z. Liu, K. Bi and X. Chen, RSC Adv., 2015, 5, 59591-59602.

30 L. Gu, X. Wang, Z. Liu, P. Ju, L. Zhang, Y. Zhang, B. Ma, K. Bi and X. Chen, Food Chem. Toxicol., 2014, 68, 226-233.

31 S. Li, M. Zhang, P. Hou, R. Zhang, C. Hou, K. Bi and X. Chen, RSC Adv., 2015, 5, 77689-77698.

32 G. Philippe, L. Angenot, M. Tits and M. Frédérich, Toxicon, 2004, 44, 405-416.

33 F. Liu, X. Wang, X. Han, X. Tan and W. Kang, Int. J. Biol. Macromol., 2015, 77, 92-98.

34 Y. Liu, X. Liang and Y. Tang, Shandong J. Tradit. Chin. Med., 2014, 33, 1014-1016.

35 Y. Tang, Y. Liu and X. Liang, Shandong J. Tradit. Chin. Med., 2014, 33, 660-662.

36 X. S. Gu, F. Wang, C. Y. Zhang, C. J. Mao, J. Yang, Y. P. Yang, S. Liu, L. F. Hu and C. F. Liu, Neurochem. Res., 2016, 1-14.

37 Z. Y. Hu, G. Liu, X. R. Cheng, Y. Huang, S. Yang, S. Y. Qiao, L. Sun, W. X. Zhou and Y. X. Zhang, Exp. Gerontol., 2012, 47, 14-22.

38 N. Y. Tang, C. H. Liu, C. T. Hsieh and C. L. Hsieh, Am. J. Chin. Med., 2010, 38, 51.

39 J. Liu, D. Z. Jin, L. Xiao and X. Z. Zhu, Brain Res., 2006, 1089, 162-170.

40 H. Y. Yu, M. G. Liu, D. N. Liu, G. W. Shang, Y. Wang, C. Qi, K. P. Zhang, Z. J. Song and J. Chen, Pharmacol., Biochem. Behav., 2007, 88, 131-140.

41 R. Sun, K. Wang, D. Wu, X. Li and Y. Ou, Folia Neuropathol., 2012, 3, 270-276.

42 C. Hou, R. Zhang, K. Zhang and X. Chen, Metab. Brain Dis., 2017, 32, 2033-2044.

43 H. Shi, C. Hou, L. Gu, H. Xing, M. Zhang, L. Zhao, K. Bi and X. Chen, Metab. Brain Dis., 2017, 32, 133-145.

44 J. Müller and K. Wanke, Fortschr. Neurol. Psyc., 1998, 66, 289295.

45 S. Li, Y. Chu, R. Zhang, L. Sun and X. Chen, Nutrients, 2018, 10, 514 .

46 U. D. o. Health and F. Human Services, Fed. Regist., 2001, 66, 206-207.
47 X. Jia, W. Li, J. Li and B. Cai, China J. Chin. Mater. Med., 2009, 34, 2396-2399.

48 J. Chen, T. Hou, Y. Fang, Z. P. Chen, X. Liu, H. Cai, T. L. Lu, G. J. Yan and B. C. Cai, Yakugaku Zasshi, 2011, 131, 721-729.

49 M. E. Pesce, X. Acevedo, D. Bustamante, H. E. Miranda and G. Pinardi, Pharmacol. Toxicol., 2000, 87, 116.

50 G. Levi, G. Bernardi, E. Cherubini, V. Gallo, M. G. Marciani and P. Stanzione, Brain Res., 1982, 236, 121-131.

51 K. Schmid, G. Böhmer and K. Gebauer, Respir. Physiol., 1991, 84, 351.

52 E. Basar and B. Guntekin, Brain Res., 2008, 1235, 172-193.

53 P. Brambilla, J. Perez, F. Barale, G. Schettini and J. C. Soares, Mol. Psychiatry, 2003, 8, 721-737, 715.

54 A. E. Calogero, Ann. N. Y. Acad. Sci., 2010, 771, 31-40.

55 C. M. Daimon, P. Chirdon, S. Maudsley and B. Martin, Am. J. Alzheimers. Dis., 2013, 1, 783-789.

56 C. D. Sladek and J. R. Kapoor, Exp. Neurol., 2001, 171, 200209.

57 M. P. Mattson, Excitotoxicity, John Wiley \& Sons, Ltd, 2017.

58 M. Mishima, Y. Tanimoto, Z. Oguri and H. Yoshimura, Drug Metab. Dispos., 1985, 13, 716-721.

59 L.-N. Gao, Y. Zhang, Y.-L. Cui and O. M. Akinyi, Evid.-Based Compl. Alt., 2015, 2015, 1-7.

60 M. Lv, M. Zhang, Y. Cheng, K. Zhang, C. Hou and X. Chen, Evid-Based Compl. Alt., 2017, 2017, 1-9.

61 A. Chen, H. Wang, Y. Zhang, X. Wang, L. Yu, W. Xu, W. Xu and Y. Lin, Int. J. Mol. Med., 2017, 40, 825-833.

62 N. Luo, Z. Li, D. Qian, Y. Qian, J. Guo, J. A. Duan and M. Zhu, J. Chromatogr. B: Anal. Technol. Biomed. Life Sci., 2014, 963, 29-39.

63 D.-Z. Liu, K.-Q. Xie, X.-Q. Ji, Y. Ye, C.-L. Jiang and X.-Z. Zhu, Br. J. Pharmacol., 2005, 146, 604-611.

64 S. Jin and B. B. Fredholm, Eur. J. Pharmacol., 1997, 329, 107. 65 K.-S. Huang, J.-G. Lin, H.-C. Lee, F.-J. Tsai, D.-T. Bau, C.-Y. Huang, C.-H. Yao and Y.-S. Chen, Evid-Based Compl. Alt., 2011, 2011, 1-8. 